\title{
An improved Fixed Step-size Adams Method Based On Taylor Extrapolation
}

\author{
He Ai-lin \\ Institute of Electronic Engineering \\ Naval University of Engineering \\ Wuhan Hubei, China \\ heailin@yeah.net \\ Xu Kai \\ Institute of Electronic Engineering \\ Naval University of Engineering \\ Wuhan Hubei, China \\ 992643156@qq.com
}

\author{
Yang Hai-liang \\ Institute of Electronic Engineering \\ Naval University of Engineering \\ Wuhan Hubei, China \\ 24011202337@qq.com
}

Bao Kai

Institute of Telecommunication Engineering

Navy Submarine College

Qingdao Shandong, China

1005356887@qq.com

\begin{abstract}
As to the numerical solution of ordinary differential equations, this paper introduces a new technique to improve the precision of the PECE form of the Adams method, which calculates the estimation of the local truncation error in every single integral step by using Taylor extrapolation. Through proper linear combinations of predicted value and corrected value, the most of the truncation error will be diminished. Moreover, a detailed theoretical derivation is also proposed, and the general laws of the integration equations' coefficients with different orders are given in tabular form, which facilitates the engineering practice. Finally, specific single differential equation and satellite orbit two-body motion equations are taken as examples to test this improved method, and stimulation results indicates that the improved Adams method can achieve a higher computational accuracy, nearly one order of magnitude, when compared with the unimproved method, which proves the effectiveness and efficiency of our proposed method .
\end{abstract}

Keywords-Taylor extrapolation; fixed-step; computational accuracy; truncation error; linear combinations

\section{INTRODUCTION}

Whether the calculation of hitting equations for ship fire control system, or the calculation of orbital dynamic equations for orbit determination, the calculation of differential equations is involved. Because of various factors, the expression of these differential equations is extremely complicated. The overwhelming majority of these equations cannot gain accurate analytical solutions other than some special situations like the two-body motion of aircraft, the computer programming is the only effective way to deal with these equations which can get approximate solutions, so seeking for these numerical methods suitable for programming is becoming increasingly important to the solution of differential equations[1].
There are some ways to improve the precision of Adams method with the same initial value, such as using higher order method, properly increase integral step-size, or using variable-step-size method [2]. However, higher order may cause instability [3], smaller integral step-size can lead to bigger round-off error, and variable-step-size method will increase the difficulty in computer implementation[4].

The estimation of truncation error is the most important part of this improved method, Fu -Zhaoping presented a detailed derivative process of the PECE form of the Adams method[5], Kai Diethelm and Zolotareva proposed detailed error analysis for Adams method[6][7].

This paper adapts Taylor explanation to analyze the local truncation error, which contribute to a more easily derivative process. The improved fixed-step-size method in this paper has a more easily engineering realization.

\section{THE IMPROVED ADAMS METHOD WITH FIX-STEP-SIZE}

In this section, we conduct the algorithm design for improved Adams method of PECE format. Taking the forth order Adams method as an example, we do theoretical analysis for the process of improving, other method 'process with higher order is similar [7].

For forth order Adams method, the PECE format is presented as follows:

$$
\left\{\begin{array}{l}
P: y_{n+1}^{p}=y_{n}+h / 24 *\left(55 f_{n}-59 f_{n-1}+37 f_{n-2}-9 f_{n-3}\right) \\
E: f_{n+1}=f\left(t_{n+1}, y_{n+1}^{p}\right) \\
C: y_{n+1}^{c}=y_{n}+h / 24 *\left(9 f_{n+1}+19 f_{n}-5 f_{n-1}+f_{n-2}\right) \\
E: f_{n+1}=f\left(t_{n+1}, y_{n+1}^{c}\right)
\end{array}\right.
$$

Then we do the truncation error analysis:

We analyze the truncation error of predicted equation by Taylor expansions; other higher order method has the similar process. For a given step-size, assuming all the 
cumulative integral error is zero, to find the truncation error $T_{n+1}$, use the first equation in equation(1), then do Taylor expansion at point $t_{n}$ :

$$
\begin{aligned}
T_{n+1} & =y\left(t_{n+1}\right)-y\left(t_{n}\right) \\
& -\frac{h}{24}\left[55 f\left(t_{n}, y\left(t_{n}\right)\right)-59 f\left(t_{n-1}, y\left(t_{n-1}\right)\right)\right. \\
& \left.+37 f\left(t_{n-2}, y\left(t_{n-2}\right)\right)-9 f\left(t_{n-3}, y\left(t_{n-3}\right)\right)\right] \\
& =y\left(t_{n+1}\right)-y\left(t_{n}\right)-\frac{h}{24}\left[55 y^{\prime}\left(t_{n}\right)-59 y^{\prime}\left(t_{n-1}\right)\right. \\
& \left.=37 y^{\prime}\left(t_{n-2}\right)-9 y^{\prime}\left(t_{n-3}\right)\right] \\
= & \frac{h^{5}}{120} y^{(5)}\left(t_{n}\right)+\frac{49 h^{5}}{144} y^{(5)}\left(t_{n}\right)+o\left(h^{6}\right) \\
= & \frac{251 h^{5}}{720} y^{(5)}\left(t_{n}\right)+o\left(h^{6}\right)
\end{aligned}
$$

As to corrected equations, after the similar process, the truncation error can be got:

$$
\begin{aligned}
T_{n+1}= & y\left(t_{n+1}\right)-y\left(t_{n}\right) \\
& -\frac{h}{24}\left[9 f\left(t_{n+1}, y\left(t_{n+1}\right)\right)+19 f\left(t_{n}, y\left(t_{n}\right)\right)\right. \\
& \left.-5 f\left(t_{n-1}, y\left(t_{n-1}\right)\right)+f\left(t_{n-2}, y\left(t_{n-2}\right)\right)\right] \\
= & y\left(t_{n+1}\right)-y\left(t_{n}\right)-\frac{h}{24}\left[9 y^{\prime}\left(t_{n+1}\right)+19 y^{\prime}\left(t_{n}\right)\right. \\
& \left.-5 y^{\prime}\left(t_{n-1}\right)+y^{\prime}\left(t_{n-2}\right)\right] \\
= & \frac{h^{5}}{120} y^{(5)}\left(t_{n}\right)-\frac{5 h^{5}}{144} y^{(5)}\left(t_{n}\right)+o\left(h^{6}\right) \\
= & \frac{-19}{720} h^{5} y^{(5)}\left(t_{n}\right)+o\left(h^{6}\right)
\end{aligned}
$$

So, we can get the following equations,

$$
\left\{\begin{array}{l}
y\left(t_{n+1}\right)-y_{n+1}^{p} \approx \frac{251}{720} h^{5} y^{(5)}\left(t_{n}\right)+o\left(h^{6}\right) \\
y\left(t_{n+1}\right)-y_{n+1}^{c} \approx-\frac{19}{720} h^{5} y^{(5)}\left(t_{n}\right)+o\left(h^{6}\right)
\end{array}\right.
$$

Where $y\left(t_{n+1}\right)$ is the value without truncation error.

Ignoring the infinitely small quantity of higher order $o\left(h^{6}\right)$,

$$
\left\{\begin{array}{l}
y\left(t_{n+1}\right)-y_{n+1}^{p} \approx \frac{251}{270}\left[y_{n+1}^{c}-y_{n+1}^{p}\right] \\
y\left(t_{n+1}\right)-y_{n+1}^{c} \approx \frac{-19}{270}\left[y_{n+1}^{c}-y_{n+1}^{p}\right]
\end{array}\right.
$$

Obviously, when doing numerical integration, if we replace $y_{n+1}^{p}$ with $y_{n+1}^{p}+(251 / 270)\left[y_{n+1}^{c}-y_{n+1}^{p}\right], y_{n+1}^{c}$ with $y_{n+1}^{c}-(19 / 270)\left[y_{n+1}^{c}-y_{n+1}^{p}\right]$, we will get higher accuracy.

When applying this improved method in the practical programming, to the improvement of predicted equation, because we cannot get $y_{n+1}^{c}-y_{n+1}^{p}$ at current step, we use $y_{n}^{c}-y_{n}^{p} \quad$ as a replacement. So we finally get the forth improve Adams method:

$$
\left\{\begin{array}{l}
P: y_{n+1}^{p}=y_{n}+\frac{h}{24}\left(55 f_{n}-59 f_{n-1}+37 f_{n-2}-9 f_{n-3}\right) \\
M: y_{n+1}^{p}=y_{n+1}^{p}+\frac{251}{270}\left[y_{n}^{c}-y_{n}^{p}\right] \\
E: f_{n+1}^{\prime}=f\left(t_{n+1}, y_{n+1}^{p}\right) \\
C: y_{n+1}^{c}=y_{n}+\frac{h}{24}\left(9 f_{n+1}^{\prime}+19 f_{n}-5 f_{n-1}+f_{n-2}\right) \\
M: y_{n+1}^{c}=y_{n+1}^{c}-\frac{19}{270}\left[y_{n+1}^{c}-y_{n+1}^{p}\right] \\
E: f_{n+1}=f\left(t_{n+1}, y_{n+1}^{c}\right)
\end{array}\right.
$$

To $\mathrm{m}(\mathrm{m}>4)$ order improved Adams method, similarly, the truncation error of predicted and corrected equation can be expressed:

$$
\left\{\begin{array}{l}
T_{n+1}^{p}=r_{m} h^{m+1} y^{(m+1)}\left(t_{n}\right) \\
T_{n+1}^{c}=r_{m}^{*} h^{m+1} y^{(m+1)}\left(t_{n}\right)
\end{array}\right.
$$

Where the partial value of $r_{m}$ and $r_{m}^{*}$ are presented in TABLE.I[9][10], the rest of which can be calculated with:

$$
\left\{\begin{array}{l}
\lambda_{i}=1-\sum_{j=1}^{i} \frac{1}{j+1} \lambda_{i-j}, \lambda_{0}=1 \\
\lambda_{i}^{*}+\frac{1}{2} \lambda_{i-1}^{*}+\mathrm{L}+\frac{1}{i+1} \lambda_{0}^{*}=1, \lambda_{0}^{*}=1
\end{array}\right.
$$

TABLE.I Partial value of $r_{m}$ and $r_{m}^{*}$

\begin{tabular}{|c|c|c|c|c|c|c|}
\hline $\mathbf{m}$ & 4 & 5 & 6 & 7 & 8 & 9 \\
\hline$r_{m}$ & $\frac{251}{720}$ & $\frac{95}{288}$ & $\frac{19087}{60480}$ & $\frac{5257}{17280}$ & $\frac{1070017}{3628800}$ & $\frac{25713}{89600}$ \\
\hline$r_{m}^{*}$ & $\frac{-19}{720}$ & $\frac{-3}{160}$ & $\frac{-863}{60480}$ & $\frac{-275}{24192}$ & $\frac{-33953}{3628800}$ & $\frac{-8183}{1036800}$ \\
\hline
\end{tabular}

Therefore, the $y_{n+1}^{p}$ and $y_{n+1}^{c}$ 'can be replacement for $y_{n+1}^{p}$ and $y_{n+1}^{c}$.

$$
\left\{\begin{array}{l}
y_{n+1}^{p}=y_{n+1}^{p}+\frac{r_{m}}{\left|r_{m}\right|+\left|r_{m}^{*}\right|}\left[y_{n}^{c}-y_{n}^{p}\right] \\
y_{n+1}^{c} \cdot=y_{n+1}^{c}+\frac{r_{m}^{*}}{\left|r_{m}\right|+\left|r_{m}^{*}\right|}\left[y_{n+1}^{c}-y_{n+1}^{p}\right]
\end{array}\right.
$$

\section{TESTING IMPROVED METHOD}

In this section, a single specific differential equation and the aircraft two-body motion equation are used as examples to test the improved method, the initial value can be provided by Kutta-Runge method with same order [5].

\section{A. Testing single differential equation}

Using the forth order improved and unimproved Adams method to solve the (10),

$$
y^{\prime}=x e^{3 x}-2 y, \quad x \in[0,1], \quad y(0)=0
$$

Meanwhile, the rigorous analytical solution of (10) owns the following expression. 


$$
y(x)=\frac{1}{5} x e^{3 x}-\frac{1}{25} e^{3 x}+\frac{1}{25} e^{-2 x}
$$

Set the integral step-size $\mathrm{h}=0.0002$ and $\mathrm{h}=0.002$, compare the numerical solution calculated by improved and unimproved method with the analytical solution provided by (11), then calculate the integral error, and map it with MATLAB. The results are shown as below:

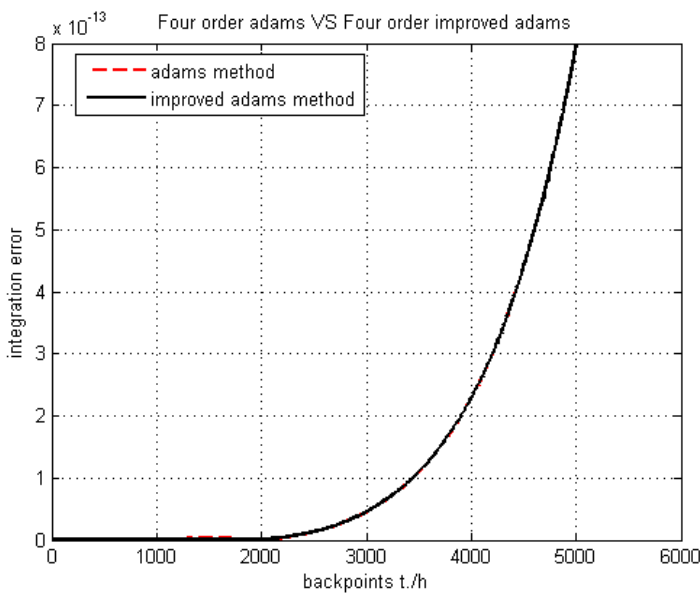

Figure 1. the change trend of fourth Adams method following the number of integration steps with $\mathrm{h}=0.0002$.

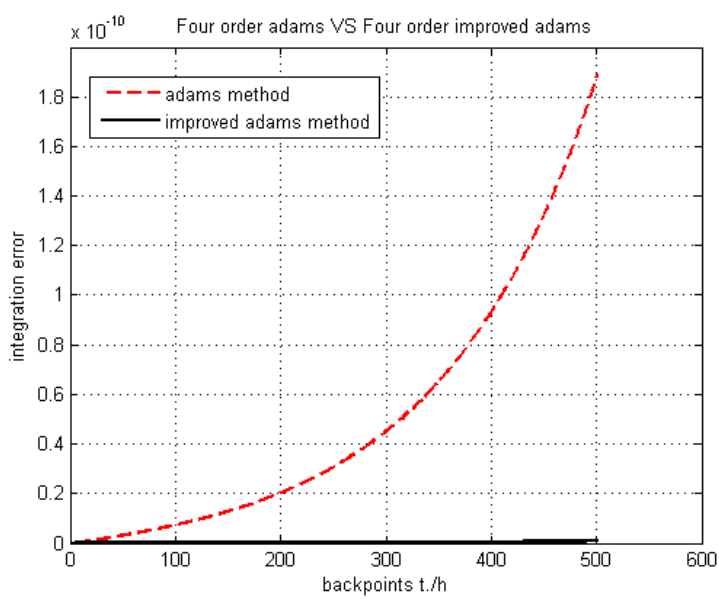

Figure .2 the change trend of fourth Adams method following the number of integration steps with $\mathrm{h}=0.002$.

From Fig .1: the improved method, when compared with unimproved method, owns almost the same change trend at smaller integration step-size. That is because round-off error is the dominant factor to integration error at lower integration step-size, while the improved method we propose in this paper mainly diminishes the effect of truncation error.

From Fig .2: the improved method, when compared with the unimproved method, owns an obvious smaller integration error at longer integration step-size, which proves the effectiveness and validity of our method.

Therefore, when computing single differential equation with computer program, we can directly use this improved method to get a higher accuracy.

\section{B. Testing satellite two-body equations}

Taking the data in TABLE.II as the initial value, we solve the aircraft two-body motion equations by using the sixth order improved and unimproved numerical method with MATLAB program.

TABLE.II Initial parameters for satellite orbit

\begin{tabular}{|c|c|c|}
\hline $\begin{array}{c}\text { Semimajor aixs } \\
/ \mathbf{k m}\end{array}$ & Eccentricity & $\begin{array}{c}\text { Inclination } \\
/ \mathbf{d e g}\end{array}$ \\
\hline 7170.99 & 0.000999 & 89 \\
\hline$X_{0} / \mathrm{km}$ & $Y_{0} / \mathrm{km}$ & $\mathrm{Z}_{0} / \mathrm{km}$ \\
\hline 3047.89 & 5404.13 & 3581.37 \\
\hline $\begin{array}{c}\text { RAAN } \\
/ \mathbf{d e g}\end{array}$ & $\begin{array}{c}\text { Argument of Perigee } \\
/ \mathbf{d e g}\end{array}$ & $\begin{array}{c}\text { Mean Anomaly } \\
/ \mathbf{d e g}\end{array}$ \\
\hline 60 & 29.9615 & 0.0384123 \\
\hline$V_{x 0} / \mathrm{km} \bullet \mathrm{s}^{-1}$ & $V_{y 0} / \mathrm{km}^{-} \bullet \mathrm{s}^{-1}$ & $V_{z 0} / \mathrm{km} \bullet \mathrm{s}^{-1}$ \\
\hline-1.96343 & -3.17517 & 6.46216 \\
\hline
\end{tabular}

The whole integration process last $24 \mathrm{~h}$, step-size: $\mathrm{h}=1 \mathrm{~s}$ and $\mathrm{h}=5: 5: 40 \mathrm{~s}, \mathrm{dx}, \mathrm{dy}, \mathrm{dz}$ represent the position error on $\mathrm{x}$ axis, $\mathrm{y}$-axis, $\mathrm{z}$-axis direction, ds is the synthesis position error, $d s=\sqrt{(d x)^{2}+(d y)^{2}+(d z)^{2}}$ unit: 1e-3m; dvx, dvy, $d v z$ represent the velocity error on $x$-axis, $y$-axis, z-axis direction, $\mathrm{dv}$ is the synthesis velocity error, $d v=\sqrt{(d v x)^{2}+(d v y)^{2}+(d v z)^{2}} \quad$ unit:1e-6m/s, the integration results within $24 \mathrm{~h}$ are shown in TABLE.III.

\begin{tabular}{|c|c|c|c|c|c|}
\hline \multirow{10}{*}{$\begin{array}{c}\text { Six order } \\
\text { improved } \\
\text { Adams } \\
\text { method }\end{array}$} & step-size & $d x$ & $d y$ & $d z$ & $d s$ \\
\hline & 1 & -0.0033 & -0.0058 & -0.0074 & 0.0099 \\
\hline & 5 & 0.0020 & 0.0035 & 0.0042 & 0.0058 \\
\hline & 10 & 0.0097 & 0.0175 & 0.0217 & 0.0295 \\
\hline & 15 & 0.1409 & 0.2551 & 0.3200 & 0.4329 \\
\hline & 20 & 1.0504 & 1.9026 & 2.3860 & 3.2274 \\
\hline & 25 & 4.9436 & 8.9546 & 11.228 & 15.189 \\
\hline & 30 & 17.471 & 31.645 & 39.675 & 53.672 \\
\hline & 35 & 51.790 & 93.635 & 112.63 & 155.35 \\
\hline & 40 & 126.53 & 229.19 & 287.24 & 388.64 \\
\hline \multirow{10}{*}{$\begin{array}{c}\text { Six order } \\
\text { Adams } \\
\text { method }\end{array}$} & step-size & $d x$ & $d y$ & $d z$ & $d s$ \\
\hline & 1 & -0.0033 & -0.0058 & -0.0074 & 0.0099 \\
\hline & 5 & -0.0023 & -0.0041 & -0.0052 & 0.0070 \\
\hline & 10 & -0.0895 & -0.1622 & -0.2060 & 0.2770 \\
\hline & 15 & -1.6049 & -2.9081 & -3.6762 & 4.9545 \\
\hline & 20 & -12.145 & -22.005 & -27.764 & 37.451 \\
\hline & 25 & -58.184 & -105.42 & -132.85 & 179.30 \\
\hline & 30 & -208.94 & -378.54 & -476.69 & 643.57 \\
\hline & 35 & -629.65 & -1138.6 & -1375.1 & 1893.1 \\
\hline & 40 & -1565.9 & -2836.9 & -3569.1 & 4820.6 \\
\hline \multirow{10}{*}{$\begin{array}{c}\text { Six order } \\
\text { improved } \\
\text { Adams } \\
\text { method }\end{array}$} & step-size & $d v x$ & $d v y$ & $d v z$ & $d v$ \\
\hline & 1 & 0.0039 & 0.0066 & -0.0072 & 0.0105 \\
\hline & 5 & -0.0023 & -0.0038 & 0.0042 & 0.0061 \\
\hline & 10 & -0.0116 & -0.0193 & -0.0116 & 0.0309 \\
\hline & 15 & -0.1680 & -0.2801 & 0.3101 & 0.4504 \\
\hline & 20 & -1.2525 & -2.0887 & 2.3117 & 3.3579 \\
\hline & 25 & -5.8953 & -9.8311 & 10.879 & 15.803 \\
\hline & 30 & -20.833 & -34.743 & 38.436 & 55.842 \\
\hline & 35 & -59.176 & -98.528 & 113.65 & 161.63 \\
\hline & 40 & -150.88 & -251.62 & 278.24 & 404.34 \\
\hline \multirow{10}{*}{$\begin{array}{c}\text { Six order } \\
\text { Adams } \\
\text { method }\end{array}$} & step-size & $d v x$ & $d v y$ & $d v z$ & $d v$ \\
\hline & 1 & 0.0039 & 0.0066 & -0.0072 & 0.0105 \\
\hline & 5 & 0.0027 & 0.0045 & -0.0049 & 0.0072 \\
\hline & 10 & 0.1068 & 0.1781 & -0.1994 & 0.2879 \\
\hline & 15 & 1.9143 & 3.1914 & -3.5592 & 5.1495 \\
\hline & 20 & 14.486 & 24.151 & -26.886 & 38.936 \\
\hline & 25 & 69.394 & 115.70 & -128.66 & 186.43 \\
\hline & 30 & 249.19 & 415.49 & -461.69 & 669.25 \\
\hline & 35 & 719.45 & 1197.7 & -1387.1 & 1968.9 \\
\hline & 40 & 1867.6 & 3114.0 & -3456.9 & 5013.5 \\
\hline
\end{tabular}


From TABLE.3: using the same longer step-size, the improved method, when compared with unimproved method, owns a nearly one order of magnitude smaller position error; this is the same to velocity error, which proves superiority and rationality of our improved method.

When using a smaller step-size such $\mathrm{h}=1 \mathrm{~s}$ in TABLE.III, we can get the same error value between these two methods, so we must chose the proper step-size in practical engineering practice, which can be obtained by digital tentative method, if we want to get a higher accuracy.

When we need to predict the satellite positon in a long time such as one week, we usually adopt a bigger integral step-size. From the data in TABLE.III, we can see that this six order improved method can achieve a small error when compared with this unimproved one, then this six order improved method will be a good choice.

\section{CONCLUSION}

In this paper, the testing result of both single differential equation and satellite orbit motion equations have proved the effectiveness of this improve method.

However, as to aircraft motion equations, we neglect the influence of orbit perturbation factors, such as atmospheric drag, the earth non-spherical shape, solar radiation pressure as well as lunar and solar perturbations and so on, we can use it to dissolve the orbit motion equations of high orbit aircraft whose perturbations is too small to calculate, when compared with earth gravity.

For practical engineering applications, these factors will bring errors if ignored, especially to low orbit satellite orbit prediction. Therefore, further study on orbit motion equations with perturbations is needed; the stability and effectiveness of our improved method need further verification.

At the same time, when testing satellite orbit motion equations, just six order methods are shown; the testing of the higher order method will be a major part of our future work. In some applications, the timeliness of numerical method is a quite important factor, so in our further study, we must take the overall program's execution time.

\section{ACKNOWLEDGMENT}

The authors wish to express their gratitude to Professor Liu of Naval University of Engineering for offering valuable advices and giving satellite orbit determination examples.

Meanwhile, without the funding from the department of navy communication, we could not finish our study in time.

\section{REFERENCES}

[1] [1]Zhang Yuxiang. Satellite Orbit Determination[M]. National Defense Industry Press, 2007.

[2] [2] Manuel Calvo,Jesús Vigo-Aguiar. A note on the step size selection in Adams multistep methods. Numerical Algorithms,2001,27(4):359-366.

[3] [3] Zhang Qiang, Liu Lin. The comparison of Adams-Cowell method and KSG method. Publications of Purple Mountain Observatory, 1998,17(1):19-26.

[4] [4] Zahari Zlatev.Stability properties of variable step-size variable formula methods.Numeische Mathematik,1978,31(2):175-182.

[5] [5]Fu Zhaoping, Li Hong. An improved Adams-Cowell Algorithm[J].Chinese Space Science And Technology,2006,2(1):22-26.

[6] [6]Kai Diethelm, Neville J. Ford, Alan D. Freed. Detailed Error Analysis for a Fractional Adams Method. Numerical Algorithms, 2004,36(1):31-52

[7] [7] N. D. Zolotareva. An estimate for the global error of the adams method on long intervals. Differential Equations, 2006,42(7):985993.

[8] [8]Guan Zhi, Lu Jinfu. Numerical Methods, 2012.

[9] [9] Matthew M.Berry. A Variable-Step Double-Integration MultiStep Integrator[D]. Blacksburg, Virgina. April 16,2004.

[10] [10]Maury,J.L, Jr. Segal,G.P. Cowell type numerical integration as applied to satellite orbit computation[J].NASA Technical Report TM-X-63542, X-553-69-46. Apr 01,1969. 